\title{
Current Status of a NASA High-Altitude Balloon-Based Observatory for Planetary Science
}

\author{
Denise M. Varga ${ }^{1}$ \\ NASA Glenn Research Center, Cleveland, Ohio, 44135 \\ Zach Dischner ${ }^{2}$ \\ Southwest Research Institute, Boulder, Colorado 80302
}

\begin{abstract}
Recent studies have shown that progress can be made on over $20 \%$ of the key questions called out in the current Planetary Science Decadal Survey by a high-altitude balloon-borne observatory. Therefore, NASA has been assessing concepts for a gondola-based observatory that would achieve the greatest possible science return in a low-risk and cost-effective manner. This paper addresses results from the 2014 Balloon Observation Platform for Planetary Science (BOPPS) mission, namely successes in the design and performance of the Fine Pointing System. The paper also addresses technical challenges facing the new Gondola for High Altitude Planetary Science (GHAPS) reusable platform, including thermal control for the Optical Telescope Assembly, power generation and management, and weight-saving considerations that the team will be assessing in 2015 and beyond.
\end{abstract}

\section{Nomenclature}

$\begin{array}{ll}A z & =\text { Azimuth } \\ E l & =\text { Elevation } \\ H z & =\text { Hertz, cycles per second } \\ \text { mas } & =\text { milliarcseconds } \\ p p & =\text { peak-to-peak } \\ R M S & =\text { root mean square }\end{array}$

\section{Introduction}

66 JISIONS and Voyages for Planetary Science in the Decade 2013 - 2022", the current Planetary Science Decadal Survey, identifies key questions facing planetary science and outlining plans for space and ground based exploration ten years into the future. NASA has been assessing concepts for a reusable, balloon-based observatory to provide a cost-effective means of implementing some of the missions identified in the Decadal Survey, with a goal of providing repeated and rapid turnaround.

The first recent mission to demonstrate planetary science observation from a balloon, a project called BRRISON (Balloon Rapid Response for comet ISON), was flown in September 2013 in an attempt to observe comet ISON. The mission demonstrated a number of important features even though the science target was not observed due to a stowlatch anomaly. A wealth of experience for the planetary science ballooning community was gained in the rapid mission. As a follow-on to the BRRISON mission, in September 2014 a mission called BOPPS (Balloon Observation Platform for Planetary Science) was flown out of Ft. Sumner, New Mexico. BOPPS observed comets Siding Spring and Jacques, as well as a number of other targets of interest and further characterized the gondola system and instruments.

NASA is in the early stages of planning for a reusable balloon platform. The project is referred to as the Gondola for High Altitude Planetary Science (GHAPS). GHAPS is intended to build off the successes of BRRISON and BOPPS and create a platform capable of high-value science observations using a carefully designed optical telescope and precision pointing capable of flying on ultra-long duration flights (up to 100 days).

\footnotetext{
${ }^{1}$ Systems Engineer, Research and Engineering Directorate, 21000 Brookpark Road, Mail Stop 86-11.

${ }^{2}$ Software Engineer, Planetary Science Directorate, 1050 Walnut Street, Suite 300.
}

American Institute of Aeronautics and Astronautics 
This paper addresses several components of the gondola system, advancements that were made, and early results in design consideration of the GHAPS project as well as technical considerations that still remain open for the team. Specifically, three main technical areas are covered.

1) The use of a Fast Steering Mirror (FSM) in the optical path for an ultraviolet and visible light instrument is discussed. The mirror utilizes a closed-loop control system that quickly adjusts the position of the mirror in order to stabilize the light passing through to the instrument. The FSM proved to be very successful in providing image stabilization for the instrument during the BOPPS mission.

2) The paper identifies some foreseen engineering challenges that the GHAPS project will be addressing. These include items such as Optical Telescope Assembly (OTA) thermal control for infrared observations, power demands for nighttime observations (it is assumed that there will be day/night cycles for the ultra-long duration flights), and mass limitations, which ties into the third area of discussion.

3) The paper details a trade assessment that was done to assess the feasibility of different materials for the gondola's primary structure. Given the large portion of the mass that is dedicated to the gondola, it was determined that significant weight saving could be obtained by switching from conventional aluminum to a composite construction. Several different manufacturing techniques were considered and will be discussed. All of the construction materials had widely varying pros and cons (raw material cost, manufacturing cost, etc).

The remainder of this paper expands on the above and provides information regarding the current direction of the GHAPS project.

\section{Design and Performance of the BOPPS UVVis Fine Pointing System ${ }^{1}$}

A primary challenge while gathering planetary science data is the small, distant, and dim nature of targets. To get high quality images from a balloon in the stratosphere, arcsecond level pointing must be maintained while the gondola is being perturbed by stratospheric winds. These winds act on the balloon, flight train, and gondola, exciting pendulumlike dynamics. The BOPPS gondola achieved arcsecond level pointing using a three part control system highlighted in Fig. 1 below.

The gondola is decoupled from rotation in the flight train and balloon by a large reaction wheel mounted on top of the gondola. This large reaction wheel controls the yaw of the gondola. The pitch of the telescope is controlled by two axial motors acting at the telescope's center of mass. Many balloon borne gondolas, including BOPPS's predecessor gondola, the Stratospheric Terahertz Observatory (STO), use these two systems exclusively. The BOPPS coarse control system improves on the former designs by including an additional roll reaction wheel near the top of the gondola. These systems together allow for $<5$ arcsecond pointing of the telescope.

The UVVis (Ultraviolet-Visible) fine pointing system (FPS) was designed to compensate for the residual motion of the coarse pointing system, improving the overall performance by two orders of magnitude. Using STO flight data as a guide, the residual motion from coarse control was expected to be less than 5 arcseconds in Elevation (El) and Azimuth (Az), and almost all of the power at frequencies less than $1 \mathrm{~Hz}$. Because BOPPS had heritage in STO, it was assumed that the BOPPS gondola control system performance would be on the same

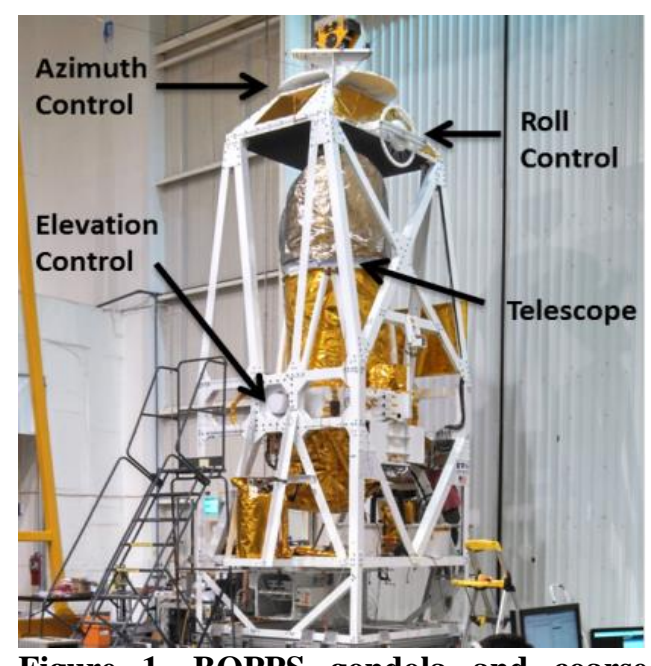

Figure 1. BOPPS gondola and coarse control components in Fort Sumner, NM. order or better with the addition of the roll compensator. Using this information the UVVis FPS was conceived with the goals of operating at $>20 \mathrm{~Hz}$ and being able to compensate for motion at $<5 \mathrm{~Hz}$. Figure 2 below shows the STO residual motion data used in the UVVis design.

The 2014 BOPPS mission had a 19 hour flight, starting at 7:30 AM on September 25th, and terminating shortly after 2:30AM on September 26th. The UVVis bench was allocated two observing windows. It is important to note that for fine pointing purposes, the BIRC (BOPPS Infrared Camera) and UVVis instruments were not intended to be used simultaneously by design. While the BIRC instrument is on, its cryopump induces vibration in the UVVis system at frequencies beyond what the UVVis FPS was required to compensate for. However, due to the long cool-down time required for the BIRC, it was not practical to shut off the cryopump in between observations. To deal with this during operations, when changes to operations and targets were being made in real-time, short datasets were obtained in a mode deemed, "cryo interruptus" where the BIRC cryopump is shut off for a short $(<60 \mathrm{~s})$ period of time. 
Characterization of the UVVis control system requires more observations over a longer period of time than can be allocated in "cryo interruptus" mode. As such, one of the two UVVis flight operational windows was intended to be mainly a focusing and calibration window, working with the BIRC bench in "cryo interruptus" mode. Encouraging pointing results were demonstrated, despite conservative correction parameters. Error! Reference source not found. depicts the reconstructed coarse gondola control residuals and post fine-steering residuals in green and blue, respectively.
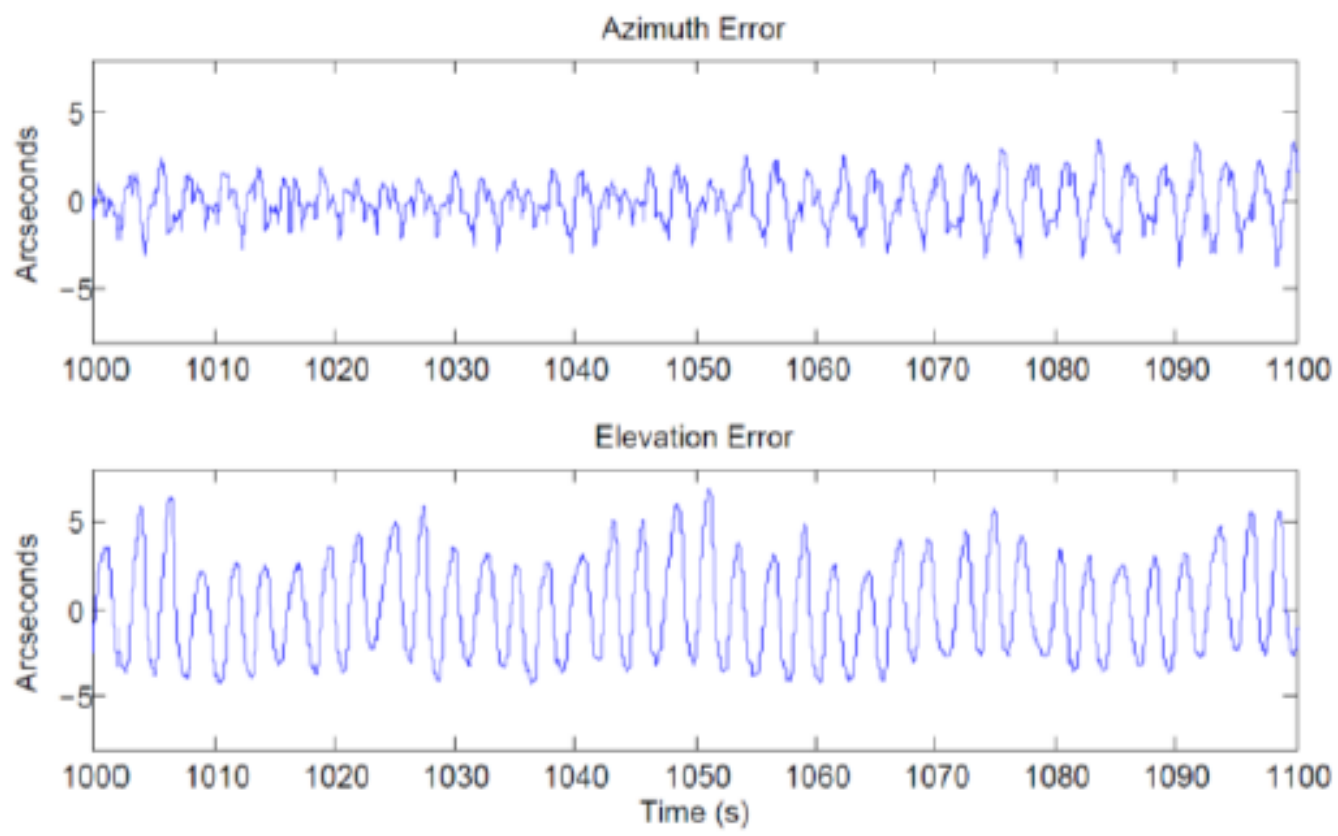

Figure 2. STO gondola residual motion is $<5$ "pp and at frequencies $<5 \mathrm{~Hz}$.

UVVis Fine Steering Performance

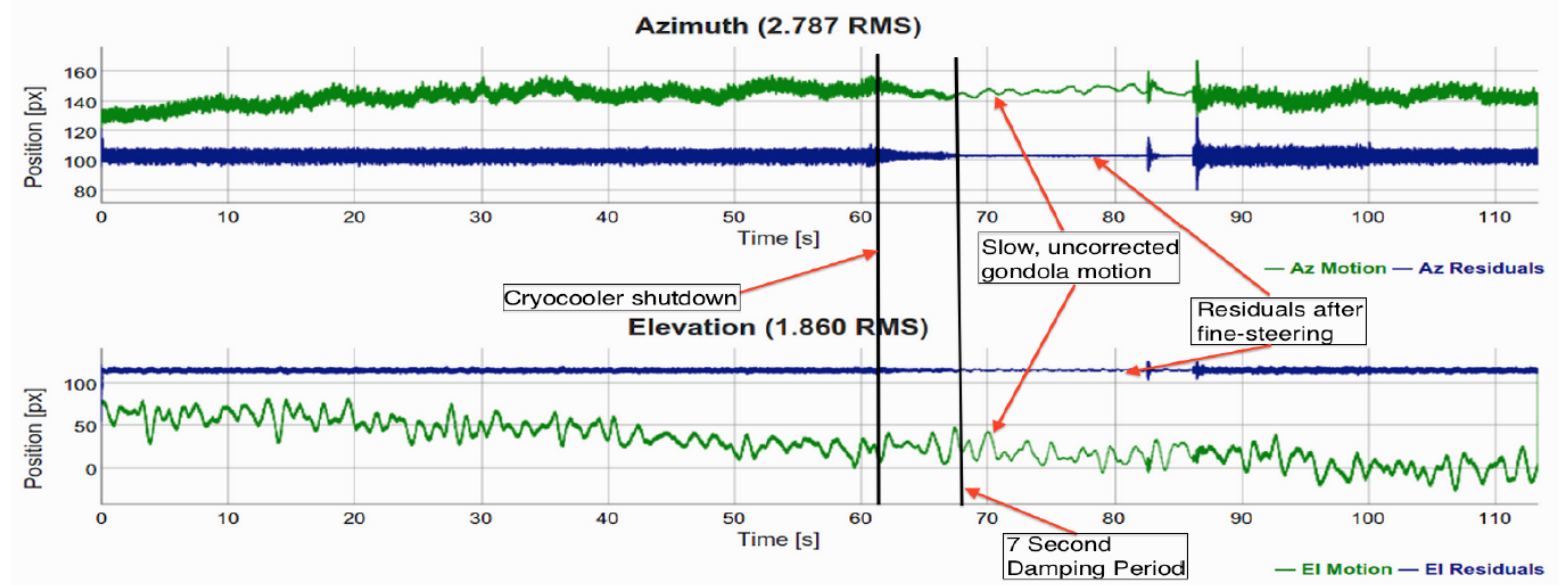

Figure 3. Fine Steering Performance Run.

At 62 seconds into the observation, the cryopump was commanded off for 20 seconds. After a 7 second damping period, the first indication of gondola motion uninfluenced by the cryopump was observed. Note that the cryopump's motion is most visible as high frequency motion on top of the slow gondola motion, and is most visible in the Azimuth direction. Since the Predictive Filter, which calculates fast steering mirror commands based on past and current error information, cutoff frequency was set to $0.5 \mathrm{~Hz}$, these high frequencies were ignored and are expected to show up in the post correction residual error. The time period of interest is between 68 and 82 seconds, where motion comes predominantly from the gondola coarse control system.

American Institute of Aeronautics and Astronautics 
Figure 4 looks only at the "cryo off" period of FPS run. In Fig. 3, the slow sinusoidal trend in the gondola residual plots (green) is absent in the post correction motion plot (blue). This illustrates the effectiveness of the UVVis finesteering system. The remaining residuals are due to a $3 \mathrm{~Hz}$ mode and motions at much higher frequencies induced by the cryopump.

Azimuth (2.787 RMS)
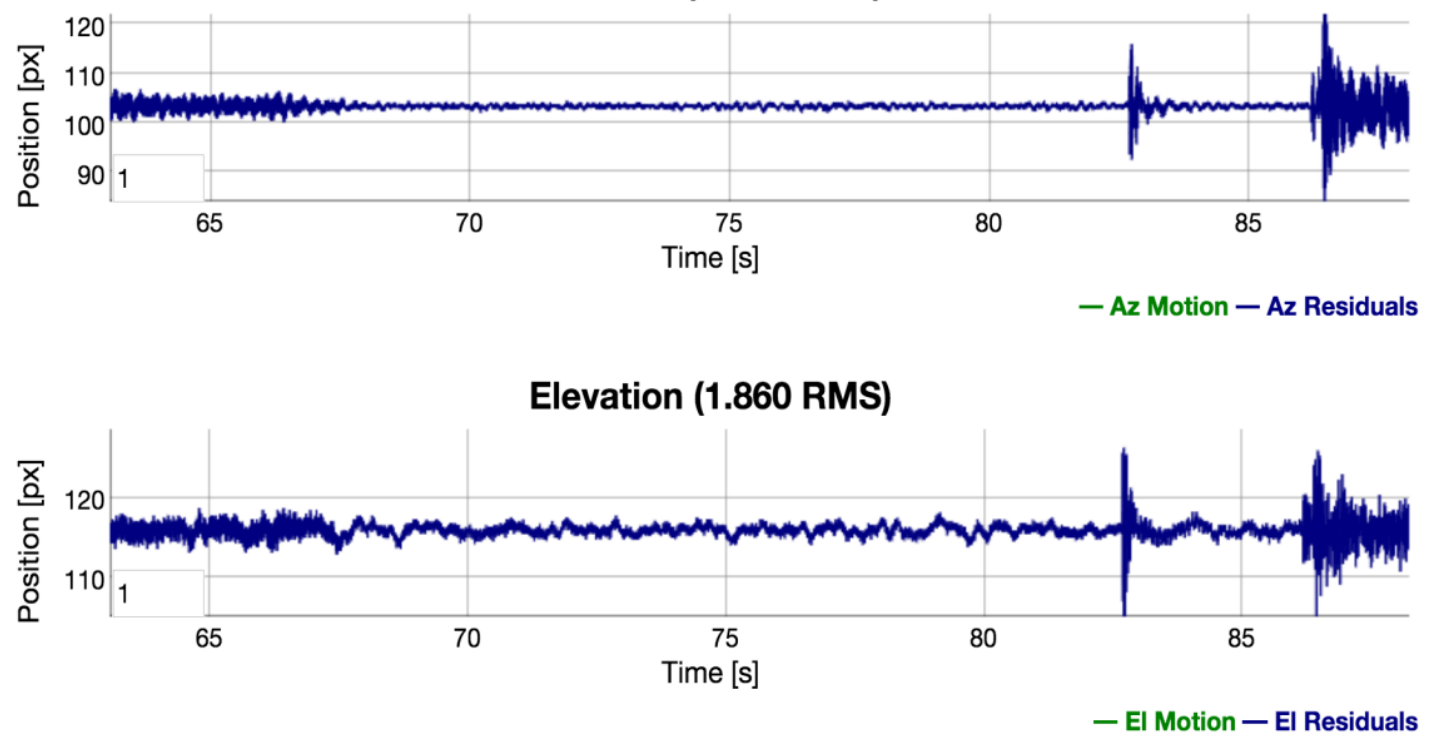

Figure 4. "Cryo off" segment of fine steering performance at $246 \mathrm{~Hz}$. 1pixel=96mas.

To quantify the pointing stability, Figs. 5 and 6 depict a rolling RMS (root mean square) calculation on the post correction residuals over the full observation period and just the "cryo off" period respectively.
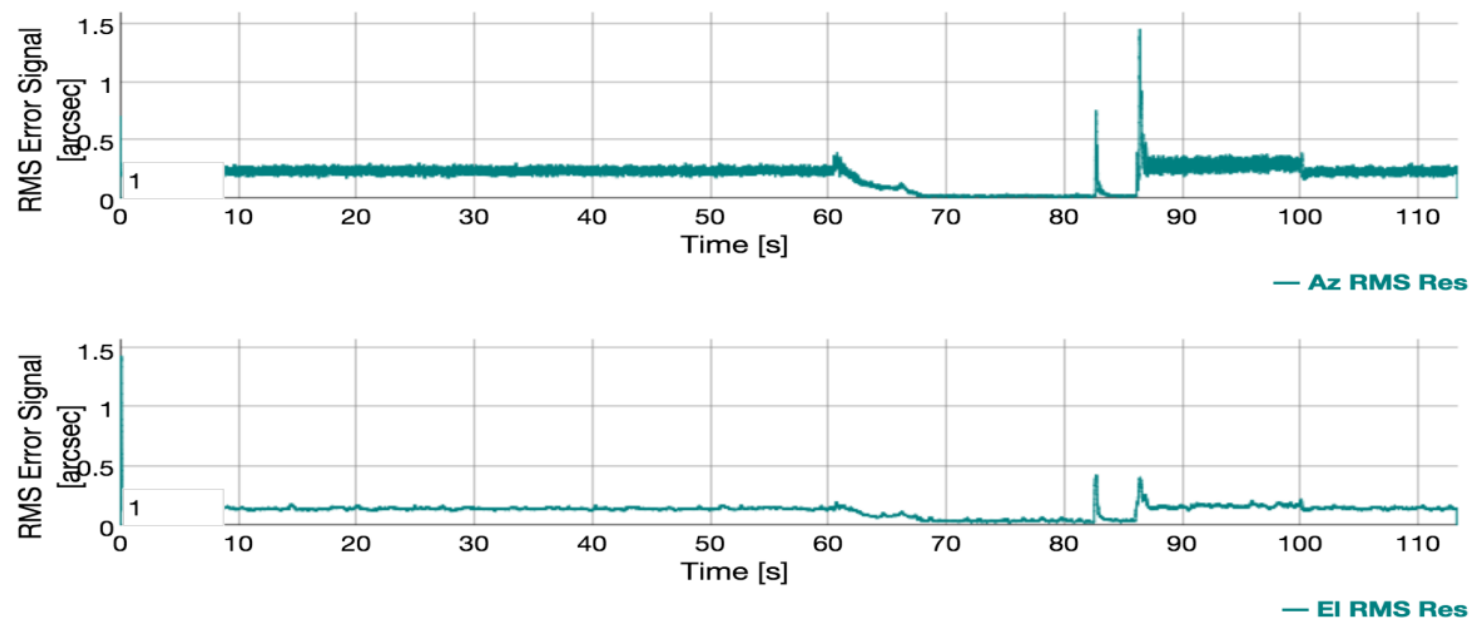

Figure 5. Fine steering residual RMS at $246 \mathrm{~Hz} .1$ pixel = 96mas. 

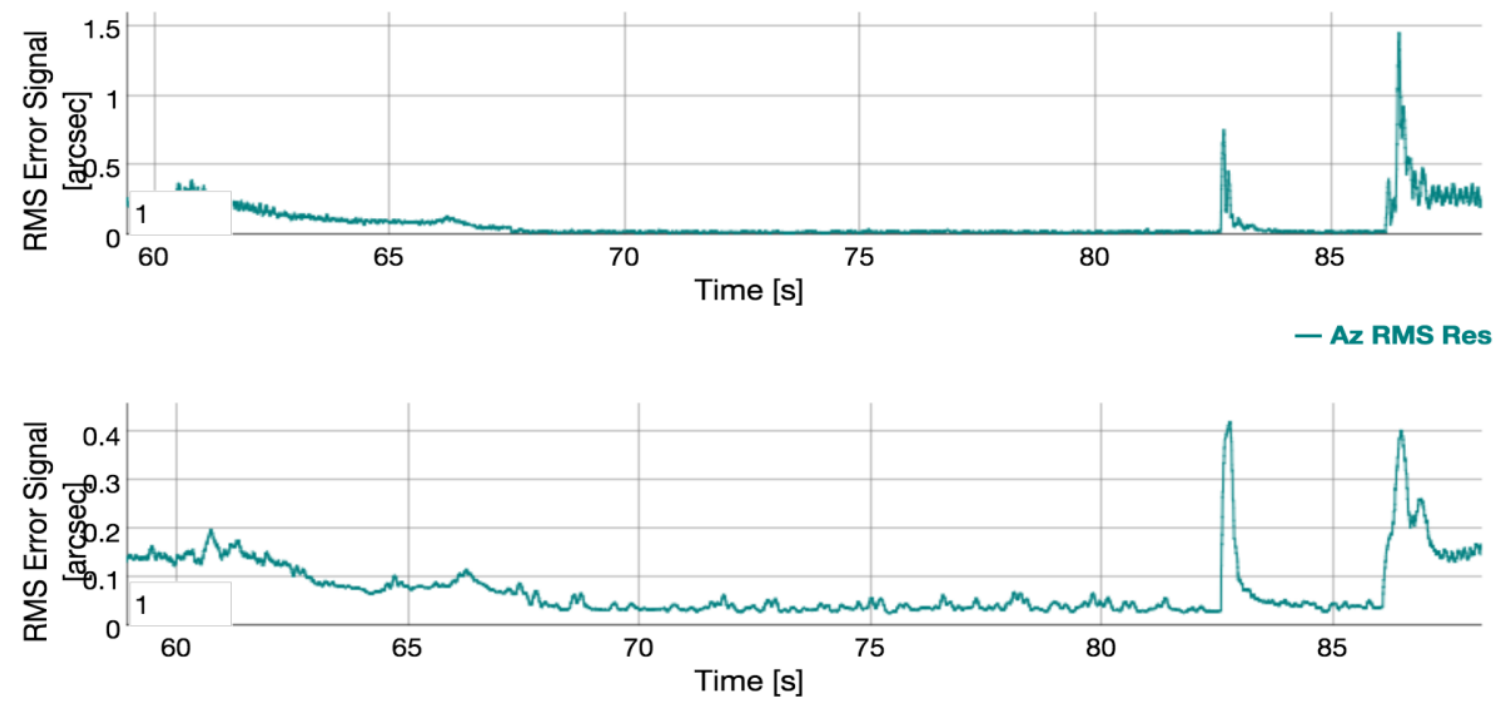

Figure 6. "Cryo-off" fine steering residual RMS at $246 \mathrm{~Hz} .1$ pixel = 96mas.

The RMS of the residual pointing error during "cryo on" periods is maintained at 280 and 165 milliarcseconds (mas) RMS for $\mathrm{Az}$ and $\mathrm{El}$ directions respectively. However, during the "cryo off" period of interest, that RMS drops dramatically. For the period of 68-82 seconds, the Az RMS error is 33.3 mas, and the El RMS error is $\mathbf{5 8 . 1}$ mas. This is a dramatic improvement over "cryo on" performance, and indicates how

Table 1. Fine Steering RMS Residuals.

\begin{tabular}{|c|c|c|}
\hline Period & $\begin{array}{c}\text { Azimuth RMS } \\
\text { [mas] }\end{array}$ & $\begin{array}{c}\text { Elevation RMS } \\
\text { [mas] }\end{array}$ \\
\hline Cryo On & 280 & 165 \\
\hline Cryo Off & 33.3 & 58.1 \\
\hline
\end{tabular}
important it is to quantify the UVVis control system without influence from the BIRC cryopump. Table 1 summarizes this comparison.

Furthermore, a closer examination of the residual motion in both directions shows motion in the 1-4 $\mathrm{Hz}$ range that the control system did not attempt to correct for in this calibration run. From testing, these motions are well within the range of the UVVis control system to attenuate while sampling at $246 \mathrm{~Hz}$. These corrections were not attempted, as at this dataset was intended as a calibration run, and operational control was given back to the BIRC science team. Test data indicates this slow, post fine steering residual motion that could have been attenuated by an order of magnitude. Without this dominating 1-4 Hz motion, even better pointing performance is expected.

\section{Engineering Considerations on the GHAPS Project}

The following section outlines several engineering considerations the GHAPS Project has started looking at in the design of the reusable gondola platform.

\section{A. Optical Telescope Assembly Thermal Control}

When making observations, particularly in the infrared spectrum, the temperature of the mirrors in the Optical Telescope Assembly greatly affects the signal to noise of images that are obtained. For this reason, keeping the mirrors as cool as possible is critical to successful science. For BRRISON and BOPPS, a sunshade was used to shield the telescope assembly from the sun. Just prior to the launch, once the launch time was known and the position of the sun relative to the observation target could be calculated, the sunshade was adjusted and fixed in place for launch. No adjustments were possible during the flight.

Long-duration missions present additional challenges to cooling the telescope assembly. During these missions there will likely be a long list of targets in different areas of the sky and their position will change over the months the platform is aloft. At launch locations near the poles (Sweden, Antarctica), there will be very short to no periods of

American Institute of Aeronautics and Astronautics 
total darkness compared to daylight hours so shielding the sun will be critical. A stationary shield may not provide the necessary protection for these longer duration missions.

A team at the Southwest Research Institute conducted an investigation into commercial-off-the-shelf shielding. First, a baffle was added around the telescope with a fixed orientation with respect to earth as in Fig. 7. Next a cylindrical radiation shield was added one inch from the outer baffle surface, Fig. 8. Then various combinations of full and partial earth and sun shields were added, Fig. 9. These were radiation panels facing in the direction of the earth or the sun. Finally, side shields were added to absorb radiation from the earth, as shown in Fig. 10.

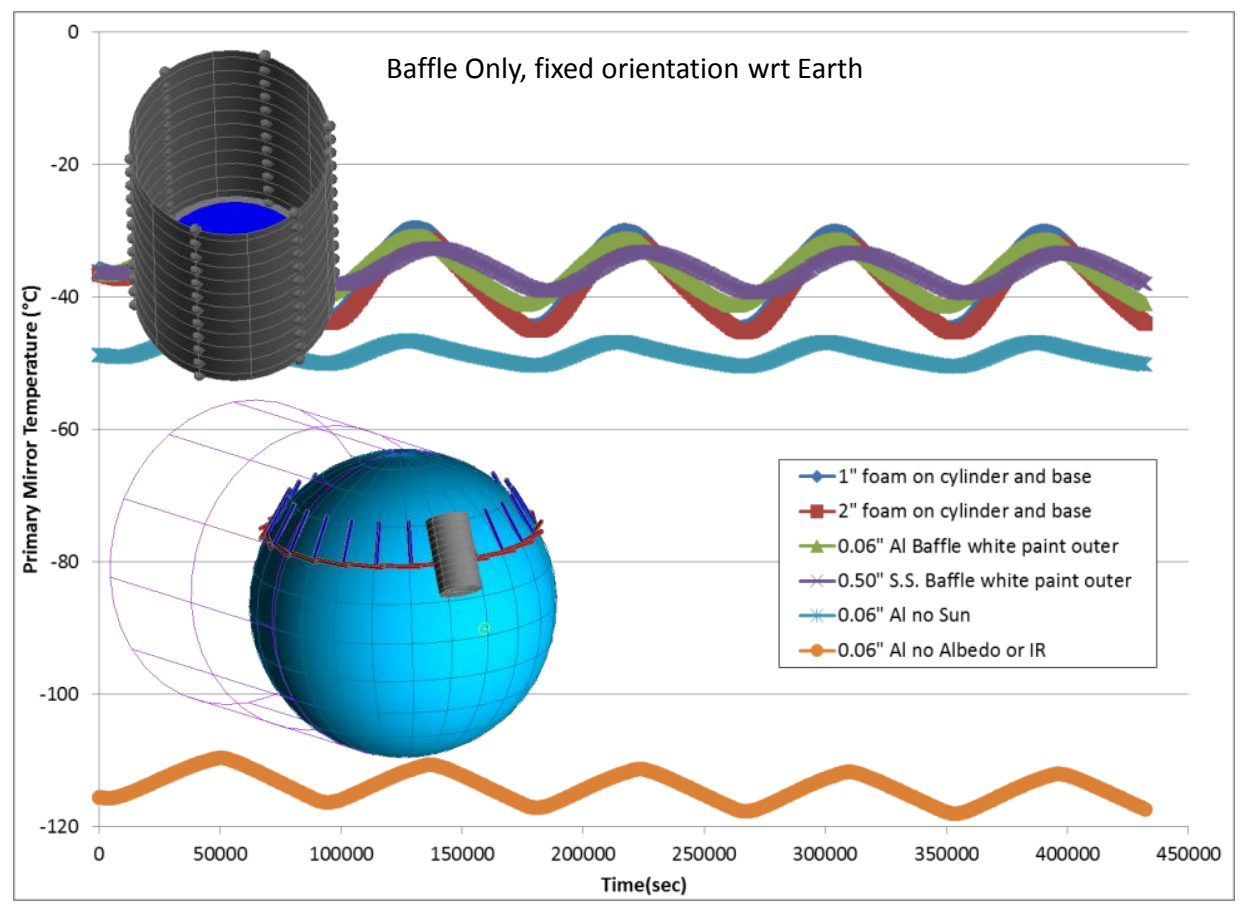

Figure 7. Baffle only.

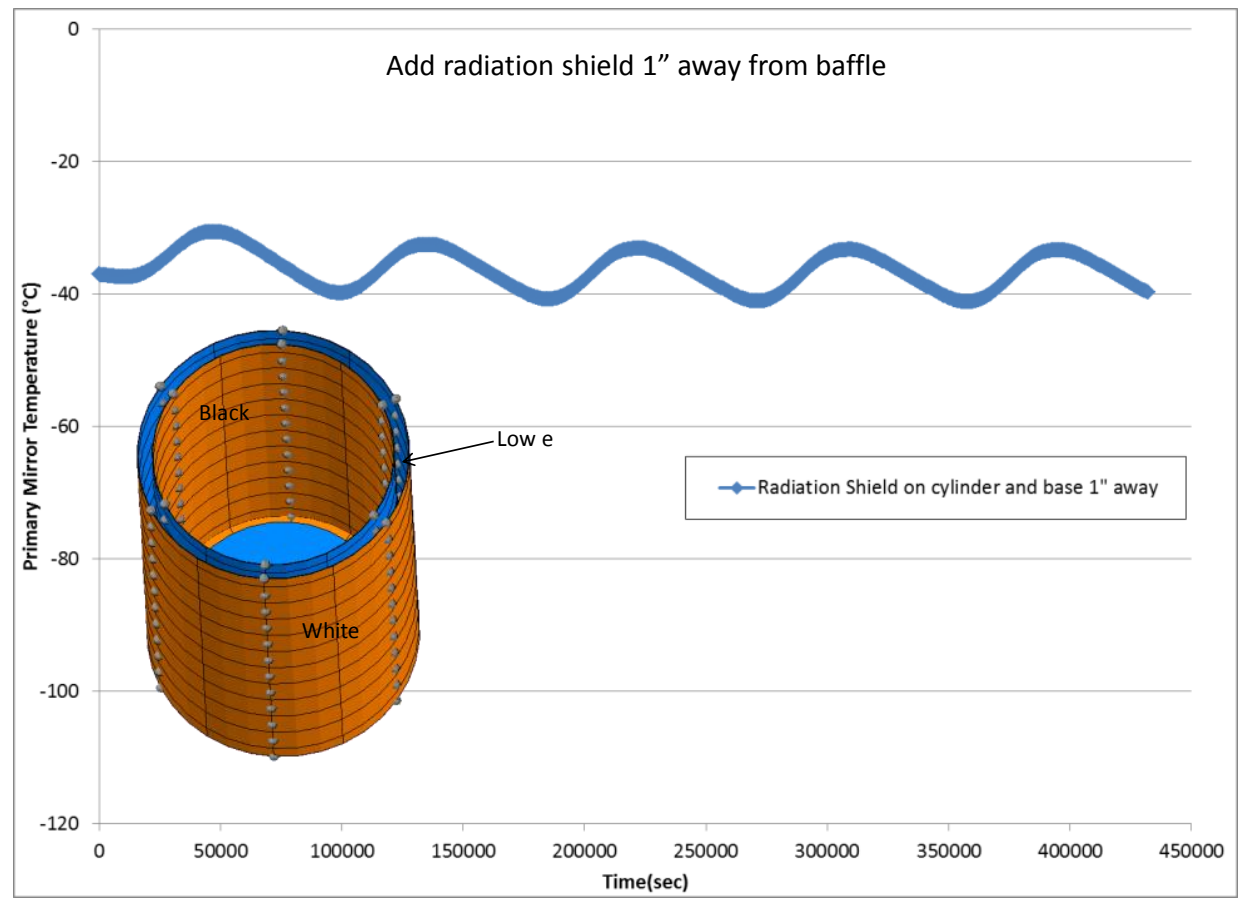

Figure 8. Baffle with radiation shield.

American Institute of Aeronautics and Astronautics 


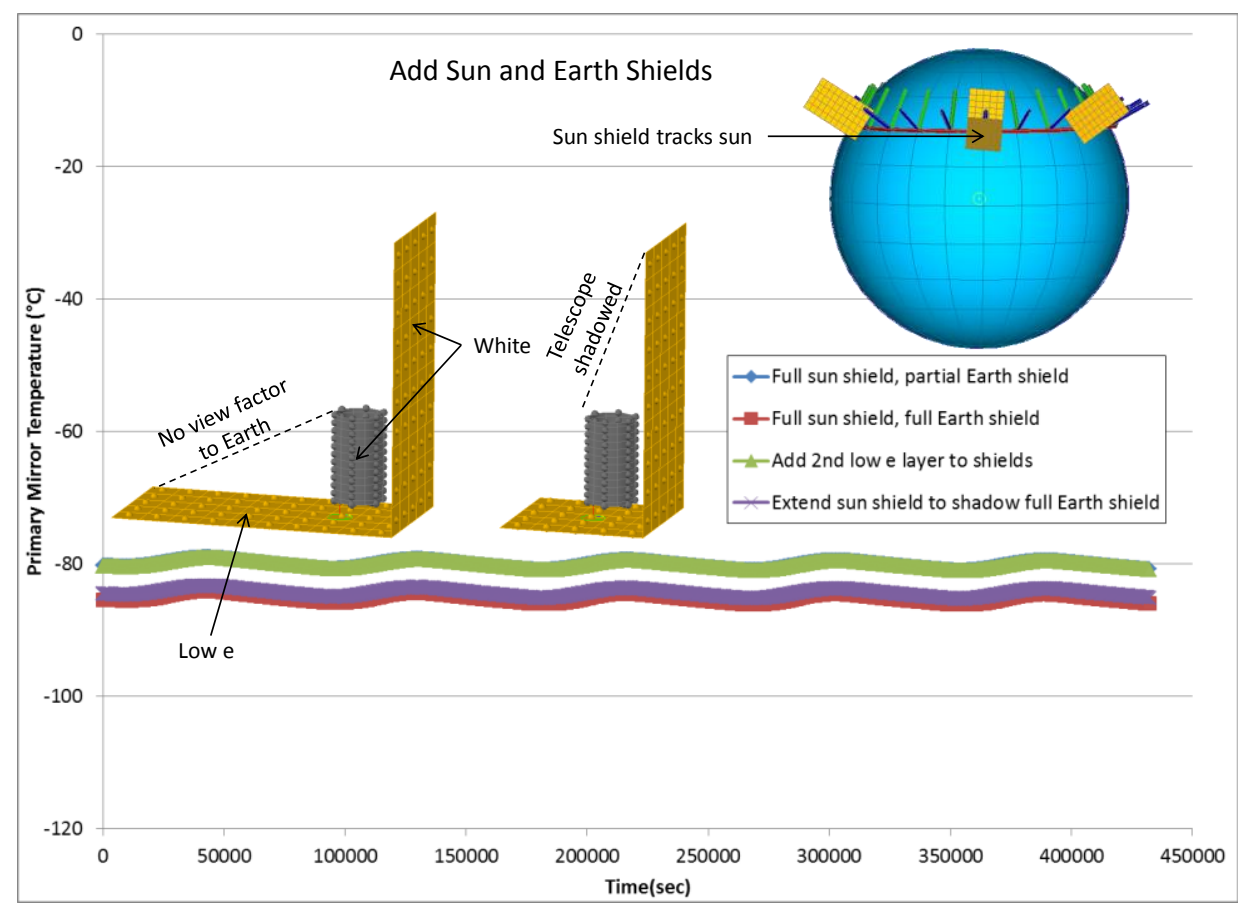

Figure 9. Baffle, radiation shield and Sun and Earth Shields.

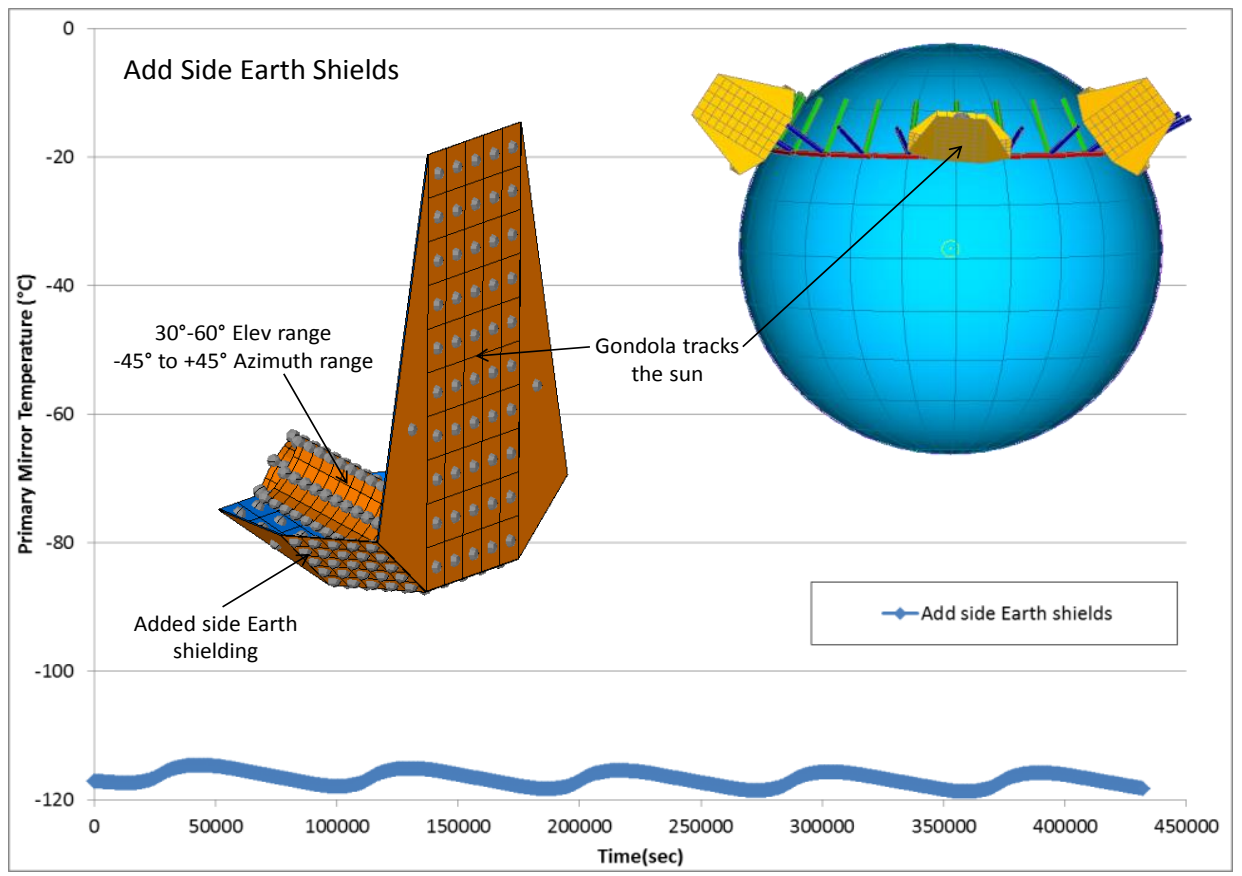

Figure 10. Thermal Protection for Optical Telescope Assembly. (With baffle, radiation shield, sun shade, and earth shades)

Several conclusions were made from this investigation.

1) Earth shield may be more important than a sun shield due to the warm infrared temperature and high emissivity on interior of baffle.

2) Earth shield still needs to be mostly shadowed to allow it to go cold and prevent reflections.

3) Additional layers of radiation shielding are only effective if there are no heat loads onto the 'cold' surfaces.

4) Design must accommodate uncontrolled orientation during ascent - sun at any angle.

5) Passive shielding looks like it might be possible for the platform.

American Institute of Aeronautics and Astronautics 
The GHAPS project will consider these conclusions during the design of the reusable gondola platform.

\section{B. Power Demands for Nighttime Observations}

Extending balloon missions from up to 24 hours, the duration for the BRRISON and BOPPS missions out of Fort Sumner, New Mexico, to four to six days (Kiruna, Sweden) or even up to 100 days (New Zealand) presents new challenges in power supply and management. For day-long missions, a battery (or bank) is sized to support the expected mission duration and when the available power drops below a certain, predefined point, the mission is terminated. There is no energy generation or replenishment on these short missions.

For long duration missions, there will be a need to generate and store power to replenish the energy supply. It is

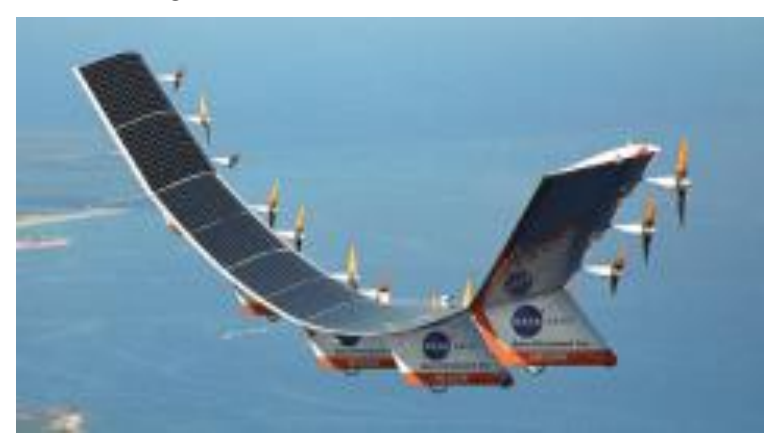

Figure 11. Example Solar Energy System. not practical to be able to fly enough batteries to support the instrumentation and data systems for 100 days and stay within allowable mass. The GHAPS team is studying solar panel/cell solutions to accommodate power generation for these long-duration missions. Recommendations based on a trade study done in October 2014 list features such as heritage with the Balloon Project Office, availability, and cost, as key considerations in a selection. Additionally, data on the performance of the cells in the mission environment is key. Finally, rigidity and fracture tolerance of the cells are important factors for re-use.

\section{Mass Limitations}

As is explained further in the next section on material selection for the gondola primary structure, mass is a critical resource for balloon flights. Both the choice of balloon type (e.g. superpressure vs conventional) and the choice of launch vehicle (each launch site has its own) will impact the allowable mass that can be carried in the gondola. Superpressure balloons offer much longer mission duration than conventional balloons, but have much lower mass capacity. In addition, the longer the mission, the greater the ballast that will need to be carried for the same type balloon.

\section{Material Selection Considerations for Gondola Primary Structure Weight Savings ${ }^{2}$}

The gondola is the structure containing all of the instrumentation and flight support equipment for a mission and takes the brunt force of landing. When designing a gondola for a reusable platform, weight is a critical consideration. While the package must be sturdy enough to support equipment and withstand flight conditions and landing, a balance must be maintained between structural integrity and gondola weight. The typical weight allowance for the gondola and flight equipment for a balloon mission is around 2,500-4,000 pounds and is determined primarily by balloon type and launch vehicle selection. Ballast is often required in addition, to assist in altitude maintenance. In order to minimize the weight of the gondola structure, and thereby maximize the weight allowance for instruments and equipment, the GHAPS team performed a trade assessment to assess light-weight materials for the gondola structure that meets the structural requirements.

Table 2. Assessment Criteria and Weighting Factor.

\begin{tabular}{|l|c|c|c|c|c|}
\hline Criteria & Mass & Material cost & Machining cost & Fabricating cost & Assembly cost \\
\hline Weight & 10 & 6 & 8 & 7 & 5 \\
\hline
\end{tabular}

Table 2 shows the criteria used to assess the proposed options and the weighting factor assigned to each. The weighting factor was assigned as a number between 1 and 10, with the criteria with higher importance receiving a higher number. Mass was identified as the most important criteria. 
Table 3. Trade Assessment Options.

\begin{tabular}{|l|l|}
\hline Option Identifier & Material and Construction \\
\hline TA-1 & All Aluminum with Semi-Monocoque Construction \\
\hline TA-2 & All Aluminum with Conventional Construction \\
\hline TA-3 & All Carbon Fiber Composite Construction \\
\hline TA-4 & Aluminum Honeycomb Core with Carbon Fiber Skin and Beams \\
\hline TA-5 & Foam Core Panels with Carbon Fiber Skin and Beams \\
\hline TA-6 & Aluminum Sandwich Panel with Carbon Fiber Beams \\
\hline TA-7 & Aluminum Skins with Carbon Fiber Beams \\
\hline
\end{tabular}

Table 3 shows the options that were chosen for consideration in the trade assessment. Seven options were formed by pairing various material configurations and construction methods. Data was gathered to identify the mass and costs for each option. Cost was gathered in several categories such as materials, machining, and assembly.

Option TA- 6 had the lowest overall cost, however the mass of TA- 6 was 2.5 times the lightest option (TA-4). In order to achieve the lightest possible mass (TA-4) there is a significant increase in material cost (ranked fifth of all options).

Based on the results of the assessment, the first choice would be TA-4, which is the Aluminum Honeycomb Core with Carbon Fiber Skin and Beams. Although this option has the second highest material costs, it has the lowest mass, combined with an assembly time that is only $15 \%$ greater than the best assembly time of 8.1 hours (for TA-7). Since assembly cost was weighted the lowest of the criteria, the impact of the greater assembly time is considered marginal.

This initial assessment will be expanded on in a future trade study on material selection for the GHAPS project.

\section{Conclusion}

Ballooning is a cost-effective method of providing science return in a rapid manner and in achieving the goals of the Planetary Science Decadal Survey. It is also an exciting and very satisfying endeavor for engineers and scientists, since the lifecycle of a mission is on the order of months to two years, rather than the five to ten years typical of planetary space missions. The BOPPS mission achieved sub-100 milliarcsecond pointing performance in a conservative test demonstration of the fine steering system. The GHAPS project is undertaking the design and construction of a reusable gondola platform with the goal of creating a capability for precision and stabile pointing and taking advantage of long duration mission opportunities. Through this project, the GHAPS team hopes to offer additional advances in observation methods and opportunities for low-cost, high- return planetary science.

\section{Acknowledgments}

D. M. Varga thanks the following who contributed to the contents of this paper: Matthew Dolloff, Tibor Kremic, Derrick Cheston, and Paul Trimarchi, from the Glenn Research Center.

\section{References}

${ }^{1}$ Diller, Jed, Dinkel, Kevin, Dischner, Zach, and Young, Elliot, "Design and Performance of the BOPPS UVVis Fine Pointing System”, IEEE Aerospace Conference 2015, Big Sky, MT, March 7-14, 2015 (submitted for publication)

${ }^{2}$ Kane, Alan, "GHAPS Materials and Construction Trade Assessment", Glenn Research Center, Cleveland, OH, October 2014 (unpublished)

American Institute of Aeronautics and Astronautics 